\title{
Pathophysiological mechanisms of blindness in facial trauma: A review*
}

\author{
André Luis Ribeiro Ribeiro ${ }^{1 \#}$, Adriana Maria Melo dos Reis ${ }^{2}$, Driene Góes Ramalho ${ }^{3}$, \\ Sérgio de Melo Alves Júnior ${ }^{4,5}$, João de Jesus Viana Pinheiro ${ }^{5}$ \\ ${ }^{1}$ Department of Oral and Maxillofacial Surgery, School of Dentistry, University Center of Pará—CESUPA, Belém, Brazil \\ ${ }^{2}$ School of Dentistry, State University of Campinas, Campinas, Brazil \\ ${ }^{3}$ Private Clinic, Macapá, Brazil \\ ${ }^{4}$ Department of Oral Pathology, School of Dentistry, University Center of Pará—CESUPA, Belém, Brazil \\ ${ }^{5}$ Department of Oral Pathology, School of Dentistry, Federal University of Pará, Belém, Brazil \\ Email: ${ }^{\text {ribeiroalr@ig.com.br }}$
}

Received 6 March 2013; revised 10 April 2013; accepted 1 May 2013

Copyright (c) 2013 André Luis Ribeiro Ribeiro et al. This is an open access article distributed under the Creative Commons Attribution License, which permits unrestricted use, distribution, and reproduction in any medium, provided the original work is properly cited.

\begin{abstract}
Blindness is a serious complication that can occur after facial trauma and may represent the loss of one of the most useful senses in the human relationship with the world-the sight. This study aims to review the pathophysiology of blindness related to facial trauma in order to identify the mechanisms by which it develops and to recognize the signs and symptoms required to establish proper diagnosis and treatment. Blindness following facial trauma may occur due to mechanisms that involve injury to the eyeball, optic nerve and eyelids. The leading causes of blindness resulting from facial trauma, and which may be altered by medical interference, are related to retrobulbar hemorrhage and traumatic optic neuropathy, which require extremely rapid diagnosis and can be accomplished with the resources available in most traumabased emergency services. The authors conclude that loss of vision tends to be irreversible in direct eyeball and optic nerve lesions, but can be prevented in retrobulbar hemorrhage and traumatic optic neuropathy. Eyeball and, especially, sight accuracy evaluation should be included in the initial care of trauma patients, so that the lesions that might lead to loss of vision are diagnosed and treated early.
\end{abstract}

Keywords: Blindness; Facial Trauma; Retrobulbar Hemorrhage; Traumatic Optic Neuropathy

\footnotetext{
"We declare that we do not have any commercial or associative interest that represents a conflict of interest in connection with the work submitted.

"Corresponding author.
}

\section{INTRODUCTION}

Blindness is a serious complication that can occur after facial trauma and may represent the loss of one of the most useful senses in the human relationship with the world - the sight. Blindness is defined as a sight loss that can physically prevent the individual from being selfsufficient in an occupation, thus making him or her dependent upon other people, agencies or apparatus to perform daily routine activities [1].

The World Health Organization (WHO) estimates there are between 27 to 35 million people with blindness all over the world today. This estimate goes up to as much as 42 million if the criterion be extended to visual accuracy of 20/200 or worse. Even where health statistics are more reliable, statistical data of people with blindness are imperfect, have a very broad variability, and can be applied according to different criteria, times and places, within a wide demographic area. Moreover, extrapolations are usually made from small samples to large populations. Data confirming lack of reliability indicate that about $90 \%$ of people with blindness in the world live in developing countries, mostly in Asia (about 20 million) and Africa (about 6 million), where large populations live in rural areas and detailed studies are limited [2].

In Brazil, there are few data on this subject. According to preliminary 2000 census by the Brazilian Statistics Institute, an estimated $14.5 \%$ of the population are people with some disability and among these, 16.6 million have some degree of visual disability, and nearly 150 thousand have declared a total sight loss [3]. According to WHO, the leading cause of blindness worldwide, after cataract, is due to impairment of the cornea resulting 
from eye trauma [4].

The incidence of blindness resulting from facial trauma has increased as a result of the increased number of traffic accidents involving cars and motorcycles. The auto industry has developed even more powerful, faster vehicles and when these are involved in accidents, the resulting higher impact forces greatly account for the occurrence of eye injuries. Other causes include injuries by firearms, assaults, domestic accidents, workplace accidents and sports trauma [5].

Blindness can develop after facial trauma through mechanisms that involve injuries of the eyelid, eyeball and optic nerve [6]. When treatment is performed at an early stage, may reverse a substantial loss in visual acuity [7]. The prognosis of trauma to the visual system is directly related to the causal agent, the type of injury and the time elapsed from diagnosis to definitive treatment. An effective method for evaluating the patient with an injury in the orbit is important in the recognition of injuries that lead to impaired visual acuity and are likely to be reversible, so it is possible to reduce the total number of victims with this type of sequel [8].

Because of the growing association between blindness and facial trauma, this study aims to review the pathophysiology of blindness related to facial trauma in order to identify the mechanisms by which it develops and to recognize the signs and symptoms required to establish proper diagnosis and treatment.

\subsection{Pathophysiology of Blindness}

Facial trauma can affect the skin, fat, muscles, nerves, and also lead to fracture of the craniofacial bones. In more severe cases, it may be associated with brain damage. The most frequent cause of fractures and severe facial injuries is related to traffic accidents. Other causes include injuries by firearms, assaults, domestic accidents, workplace accidents and sports trauma. On the face, the injuries may lead to loss of skin sensitivity, scars, retractions, breathing problems, facial paralysis, malocclusion, tooth loss and changes in sight, such as fractures involving the orbit and can lead to blindness [5]. The facial fractures mostly associated with blindness are fractures of the zygomatic complex, fronto-naso-orbito-ethmoidal fractures, Le Fort II and III maxillary fracture [9].

The blindness caused by facial trauma may result from mechanisms that include direct injury of the eyeball; direct injury to the optic nerve, such as those caused by bone fragments; indirect injury to the optic nerve, such as lacerations, rupture and stretching of its fibers; loss of eyelid integrity; poor perfusion of the optic nerve as a result of regional vascular complications, such as traumatic optic neuropathy and traumatic retrobulbar hemorrhage [10].

\subsection{Direct Eyeball Injury}

The direct injury of the eyeball can be open-type if it has a total thickness wound on the cornea wall of the eye. This can be caused by blunt trauma with rupture of the eyeball, by sharp objects (laceration or perforation, with or without foreign body entrained in the eyeball) [11].

A closed-type injury in the eye may not have a total eye wall thickness, but on a part of it, and it may include superficial lamellar laceration, foreign body, and contusion of the eyeball. Four variables are important for rating eyeball injury: the mechanism of injury; the degree of injury (determined by visual acuity), the presence or absence of a relative afferent pupillary defect; and an area of the eye the injury was involved [10].

The area in the eye where the injury can be involved is rated as: Zone I-from the cornea to the corneal limbus; Zone II-an area stretching to the back of the limbus (5 $\mathrm{mm}$ ) and includes damage to the iris, lens and ciliary body. Zone III-the entire structure behind zone II including the retina, optic nerve, choroid and the presence of a vitreous hemorrhage [12].

Ultrasound has proven useful in detecting rupture of the eyeball, the presence of foreign bodies, lacerations and retinal detachment (Figure 1). One must be careful during the examination because of the pressure applied to the eye, as it may expel ocular content even further in an open-type contusion [7].

In closed-type eyeball injuries, injuries of eyelid and subconjunctival hemorrhage may be similar to that of the open wounds on the eyeball [10].

The treatment of eyeball injuries depends on whether the injury is open-type or closed-type. Analgesic and antipyretic should be prescribed. A thick dressing should be placed on the eye to prevent patient's attempts to scrub the wound with the hand, especially in children [12].

Primary surgical repair of the eyeball in open-type wounds should be performed under general anesthesia as early as possible and not exceed more than 24 hours after the trauma. During repair, the posterior extension of the wound should be explored and closed with nonabsorbable suture. Foreign bodies retained in the globe should be removed at the same time [10].

The postoperative care should include antibiotics with ciprofloxacin or a combination of vancomycin and ceftazidina, prescribed to reduce the risk of endophthalmitis. Posterior vitrectomy is often necessary to clean the vitreous hemorrhage in order to prevent the vitreoretinopathy proliferation and consequent retinal detachment. The postoperative course aims to control inflammation, infection, pain and intraocular pressure. Closed-type eyeball injuries are treated with the anti-inflammatory steroids, antibiotics and antihypertensive eye drops $[7,10]$.

The prognosis depends on the degree of initial injury 

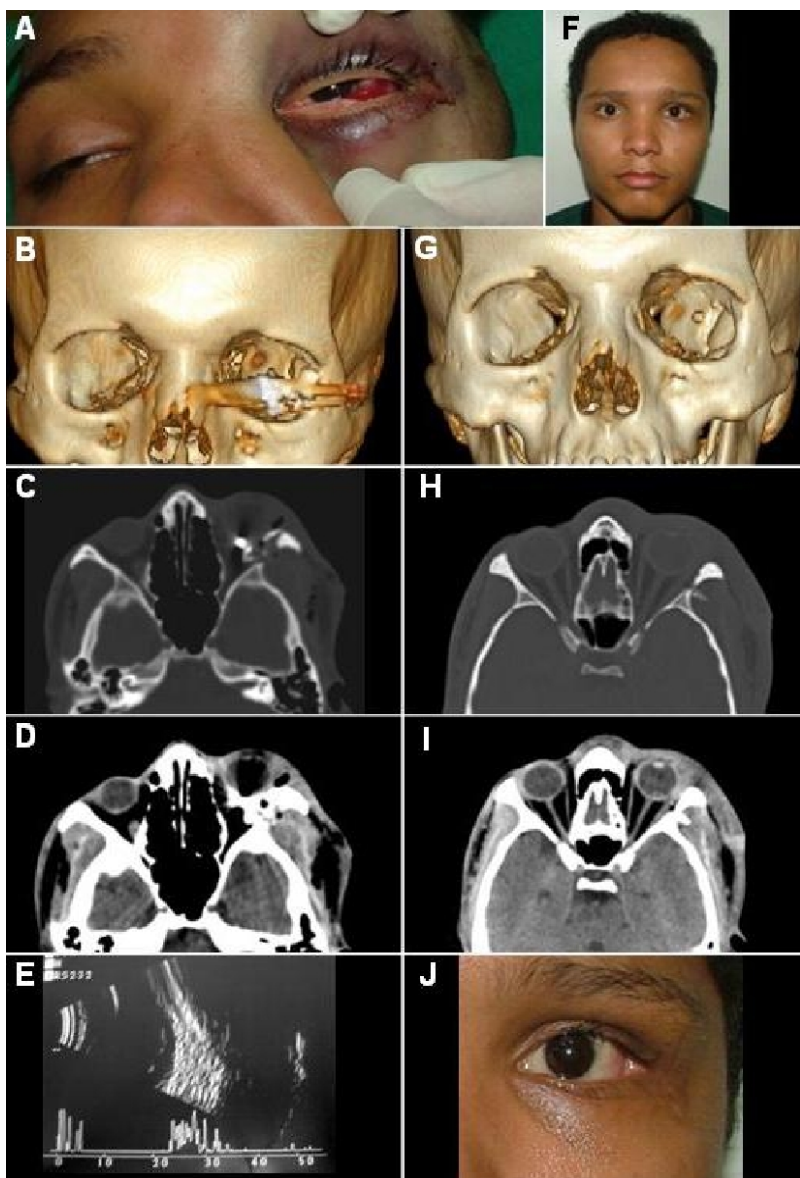

$\mathbf{H}$
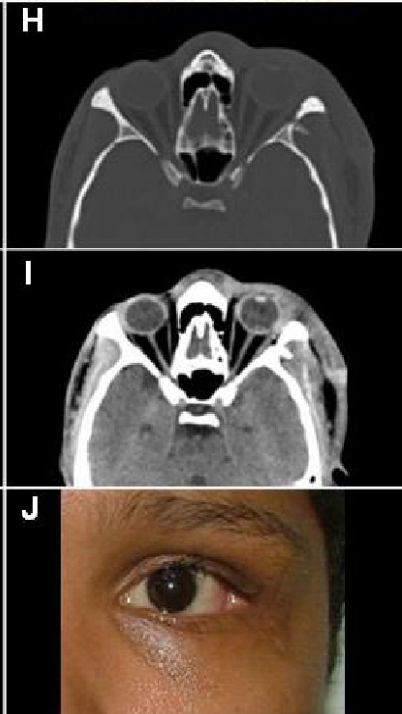

Figure 1. Patient injured by gunshot who developed blindness after blunt trauma of the eyeball due to retinal detachment. The patient underwent surgery with removal of foreign body and high doses of corticosteroids, but remains with light perception only. Initial clinical aspect (A); 3-dimension computerized tomography reconstruction (3-DCTR) with presence of foreign body in orbit (B); Axial cuts on computerized tomography (CT) showing a foreign body posterior to the eyeball, in window for bone and soft tissue, respectively (C and D); Ocular ultrasound showing a postoperative retinal detachment in the left eye (E); Postoperative facial clinical aspect showing symmetry in position and volume of eyeballs (F); 3-DCTR after surgical removal of foreign body in orbit (G); Axial cuts on CT after foreign body removal. Notice the integrity of the optic nerve without signs of bleeding or edema in the window for bone and soft tissue, respectively ( $\mathrm{H}$ and $\mathrm{I}$ ); Postoperative clinical aspect in detail of the left eye $(\mathrm{J})$; notice its integrity is maintained.

of the globe, and there may be complications such as endophthalmitis, retinal detachment, glaucoma, cataract and membrane formation on the retina [7]. In general, low visual acuity, presence of a relative afferent pupillary defect, and subsequent involvement of the eye carry a poor prognosis. This is valid both for open- and closedtype eyeball injuries $[7,10]$.

The sympathetic oftalmite is a rare complication that occurs in ocular perforations. It is characterized by uveitis in the healthy eye, which develops more than fourteen days after trauma and may lead to blindness. The early repair of the injured eye reduces the incidence of this complication and of early enucleation [13].

\subsection{Direct and Indirect Injury to the Optic Nerve}

The direct injury of the optic nerve itself is unusual. Since the nerve's trajectory is partially located inside the optic canal and this canal, because it consists of a dense bone, provides a protection to the optic nerve in fractures of that region. This makes the entire force of the trauma to spread around the foramen, thereby preventing the spread of the strength in the nerve [10] (Figure 2).

The indirect injury of the optic nerve is the most common complication of facial trauma and can be defined as part of the orbital compartment syndrome. These complications would be the result of secondary necrosis; concussion of the optic nerve caused by striking bone, with or without bleeding, partial or complete laceration of the optic nerve, interruption of the optic nerve vessel nutri-

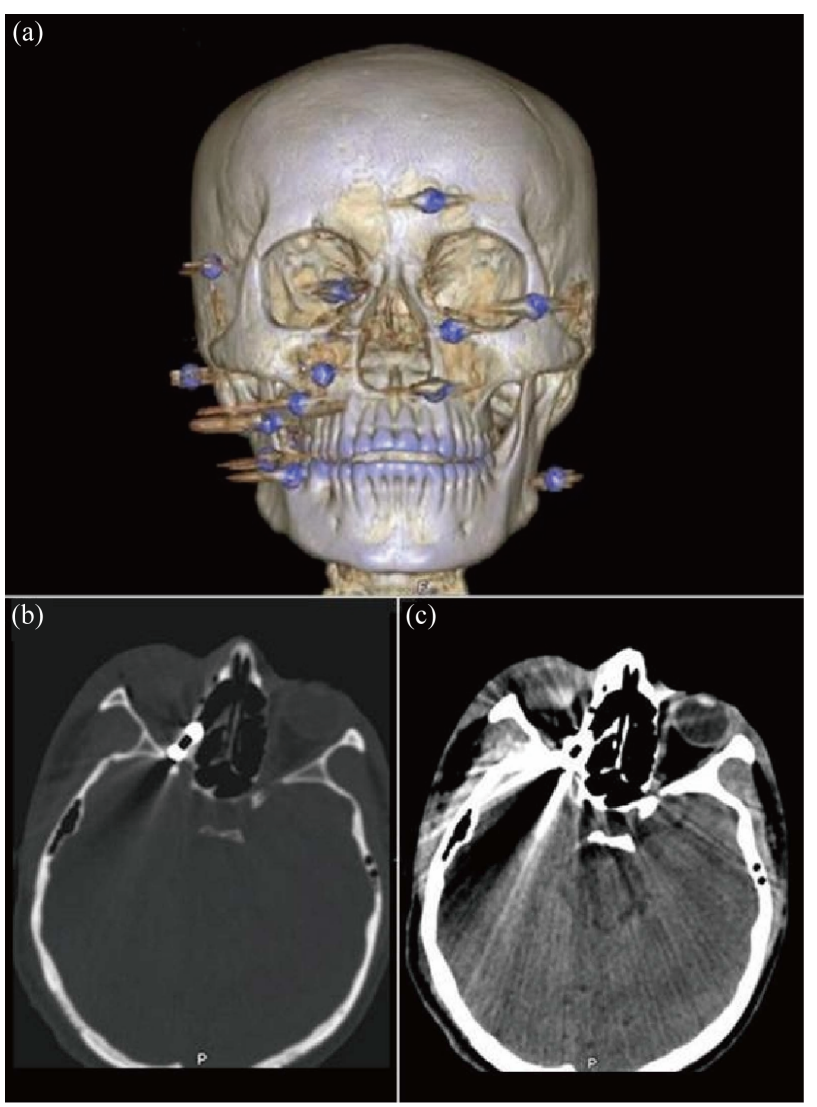

Figure 2. CT in a patient injured by gunshot with multiple projectiles (shotgun), which caused blindness by direct injury to the optic nerve of the right eye. 3-DCTR showing the distribution of multiple projectiles to the face (a); Axial cuts on CT showing two foreign bodies in orbit, one being inside the optic canal carrying a direct nerve injury in window for bone and soft tissue, respectively (b) and (c). 
tion system, hematoma formation, and vascular insufficiencies. The main causes of these complications include retrobulbar hemorrhage and traumatic optic neuropathy [9].

\subsection{Orbital Compartment Syndrome (OCS)}

The OCS is one of the few ophthalmologic surgical emergencies with which both ophthalmologists and emergency physicians should be familiar. The orbit is a closed space with limited capacity to expand. So, when OCS occurs there is an increase in volume within the orbit, resulting in an increased orbital pressure. The pathophysiology is similar to other compartment syndromes (e.g., compartment syndrome of the limbs) that are common to result in significant morbidity [6]. The OCS can lead to impaired nerve and retina, and can develop rapidly and cause blindness [14].

The etiologies of the OCS include orbital hemorrhage, which can be caused by trauma, surgery, bleeding from pre-existing injuries or other medical conditions, emphysema, cellulitis and edema in the orbit, foreign materials, such as a contrast medium or ointments, and other causes, such as allergic reactions to anesthetics [15].

The diagnosis of the syndrome is essentially clinical and a rapid and accurate diagnosis and detailed history of trauma, including signs of increased intraorbital pressure, is of great importance. Imaging tests such as CT and MRI facilitates the correct diagnosis of the injury [16].

Surgical treatment can be initiated by orbital decompression using the lateral canthotomy and cantholysis techniques, which aims to relieve tension and restore perfusion of the optic nerve and retina. Several studies show a significant and immediate decrease of orbital pressure after this simple procedure. Once the lateral canthotomy and cantholysis techniques have been performed, the clinical signs should be constantly reviewed and, if no improvement is observed within a few minutes, the orbital septum should be divided from the orbital rims. If the orbital compartment syndrome results from a post-surgical bleeding, it should be decompress through an orbital decompression surgery and then drain the hematoma and cauterize the bleeding vessels [6].

The main causes of OCS in acute trauma are the retrobulbar hemorrhage and traumatic optic neuropathy [17].

\subsection{Retrobulbar Hemorrhage (RBR)}

The RBH is characterized by bleeding in the posterior region of the orbit that leads to ischemia of the retina and optic nerve. The increased of the intra-orbital pressure is caused by the association of hemorrhage and edema. As there is increased pressure, the vessels of the retina and ophthalmic vessels are compressed, resulting in retinal ischemia [16].
The diagnosis of $\mathrm{RBH}$ is clinical and must be treated immediately after the emergency treatment $[10,16]$. Depending on the patient's overall condition and the likelyhood of recovery of sight, decompression surgery is needed immediately. If treatment is not done urgently, irreversible damage will occur after 60 minutes of ischemia [10]. The characteristics of $\mathrm{RBH}$ include decreased visual acuity, pain, ophthalmoplegia, tense proptosis, relative afferent pupillary defect, and pale optic disc [15] (Figure 3).

In patients whose visual acuity cannot be evaluated due to a low state of consciousness and, thus, incapable of reporting pain, the only evidence of the presence of $\mathrm{RBH}$ is tense proptosis and dilated pupil $[10,16]$. This

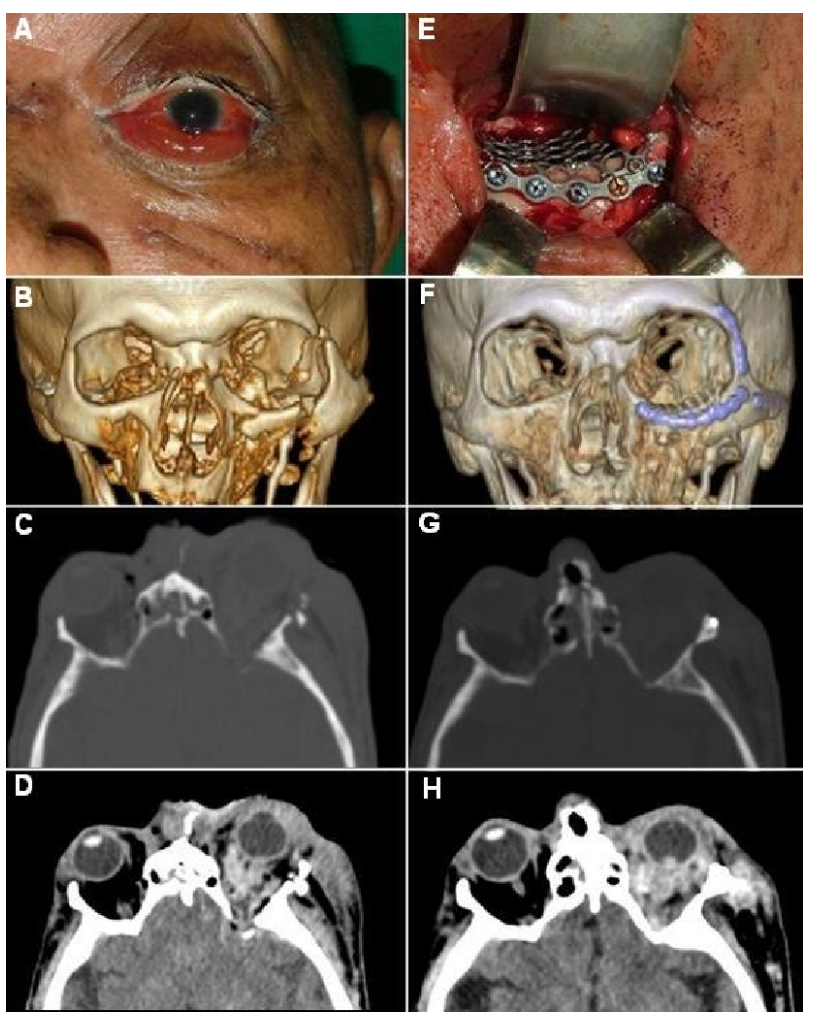

Figure 3. Patient involved in a motor vehicle accident which had a traumatic brain injury and required tracheal intubation and sedation in the emergency room. The patient developed a retrobulbar hematoma diagnosed later that evolved to blindness in the left eye. Clinical aspect of the left eye showing exophthalmos, mydriasis and subconjunctival hemorrhage (A); 3DCTR showing a fractured jaw and left zygomatic orbital (B); Axial cuts on CT showing an inflammatory posterior to the eyeball by the presence of RBH in window for bone and soft tissue, respectively (C and D); Trans-operative aspect of the orbit after attempted surgical decompression and orbital reconstruction (E); Postoperative 3DCTR after zygomatic orbital reconstruction (F); Postoperative axial cuts on CT with maintenance of the inflammatory infiltrate showing post-eyeball inflammatory infiltrate, in window for bone and soft tissue, respectively ( $\mathrm{G}$ and $\mathrm{H}$ ). The attempt to drain the hematoma was not possible, because the clot was already organized. 
diagnosis can be obtained through a careful palpation of the eyeball and, if possible, a fundoscopy. In doubtful diagnostic cases, it is important to perform CT of the skull and orbit [18].

The clinical treatment of RBH will be through medication, with use of high doses of steroids intravenously, until there is reduced pressure of the eyeball. Among these steroids, acetazolamide (250 to $500 \mathrm{mg}$ ) and mannitol (1 g/kg) may be used [7,16,18].

The primary surgical treatment of RBH can be accomplished through lateral canthotomy and cantholysis. This technique consists of an incision in the lateral eye and lateral canthal ligament section that will allow an anterior displacement of the eyeball, thus allowing for an indirect, temporary reduction of internal pressure, which in turn is performed with local anesthesia. The incision is made lateral to the corner of the orbital rim, and the tendon is identified and sectioned [19].

The definitive surgical treatment for $\mathrm{RBH}$ is done by decompression of the orbit under general anesthesia. The hematoma is drained surgically through the orbital and intraconal space. This procedure, besides improving the drainage of blood and inflammatory infiltration, allows placement of a drain to block the formation of a new hematoma [7].

An orbital hematoma can cause complications involving vascular compression and acute stretch of the optic nerve resulting from exophthalmos. The increase in retrobulbar edema may reduce the perfusion of the retina and compress the ciliary vessels, leading to ischemia of the optic nerve. Furthermore, because of the communication system, ischemia of the optic nerve may be present and lead to irreversible damage without a noticeable increase in intraocular pressure. In other situations, the sight may not necessarily be at risk, but the displacements of orbital structures by the hematoma may interfere with eye function [15].

\subsection{Traumatic Optic Neuropathy (TON)}

TON occurs when the strength dissipation of the trauma affects the optic canal area resulting in optic nerve injury. These traumas trigger an inflammatory process, releasing a cascade of chemical mediators that cause vasospasm and a secondary vaso-occlusion, edema and necrosis. This can result in neural compression leading to orbital compartment syndrome. This lesion is initially reversible, but if left untreated it will progress rapidly to vascular obstruction, causing irreversible injury [10].

The initial diagnosis for TON is clinical and the findings that suggest an optic nerve damage include decreased visual acuity and a relative afferent pupillary defect. CT scans can be used in imaging exams to help identify fractures in the optic canal and MRI can show soft tissue swelling and bruising [20].
TON requires immediate ophthalmologic consultation, and treatment should be started soon after it is detected. The treatment is controversial and may be clinical or surgical, both aiming to revert the inflammation and ischemia in the optic nerve. Clinical treatment is performed through high doses of anti-inflammatory steroids. Surgical decompression is controversial and its role is usually reserved for patients who do not respond to drug treatment, but there is still a visual recovery [10]. The lateral approach is a satisfactory decompression technique in fractures involving the lateral orbital wall. The transethmoidal approach provides direct access to the medial orbital wall fractures, eliminates the morbidity of a craniotomy, and allows for decompression of optic canal [14].

\subsection{Loss of Eyelid Integrity}

The inability to close eyelids quickly can result in corneal dryness, ulceration and blindness $[7,10]$. The avulsion of the eyelid is a rare injury, but devastating and difficult to rebuild. Moreover, lacerations of the eyelids may indicate a serious eye injury [10] (Figure 4).

Acuity and visual field, color vision and eye movement should be examined in all patients with lacerations of the eyelid. Position, length and depth of the wound must be evaluated. Lesions located medially to the eyelid can damage the lacrimal drainage system and require special attention [8].

The laceration can be repaired by gradually pulling the fragments of the eyelids to cover the cornea and, if necessary, traction suture should be used. Thus, the objective is to avoid any epithelial damage, ulceration or loss of sight. The application of topical chloramphenicol and/or artificial tears can be prescribed and the whole area covered with a wet sterile gauze pad [21].

The eyelid is a vascular tissue; even when lacerated or

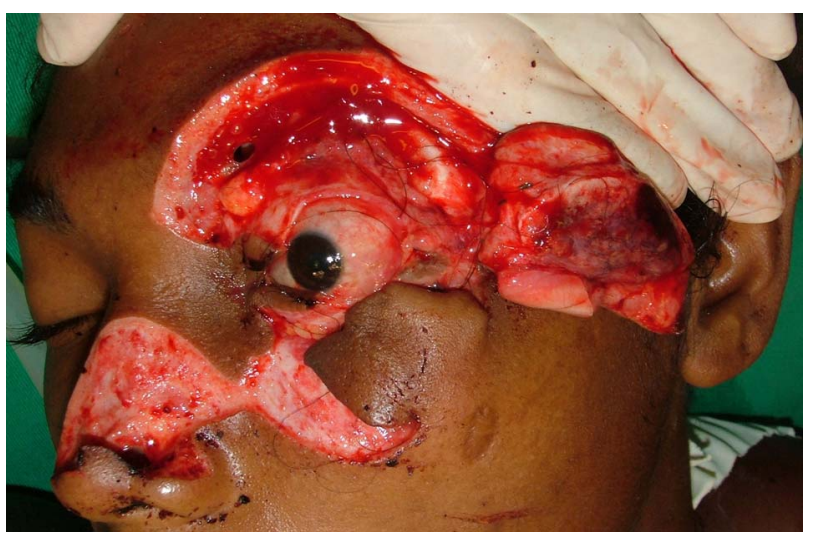

Figure 4. Patient suffered stab wound in the face involving the upper and lower eyelids. This type of injury may compromise the protection and lubrication of the eye. It requires care in reconstruction. 
necrosed, the tissue regenerates itself, so no tissue should be removed. Aesthetic and functional results can be achieved in the primary repair, but secondary reconstructions may be necessary [22].

\section{DISCUSSION}

Facial trauma is a topic of extreme importance today, as it affects mainly young adults in their productive years. Current trends are intended not only to set broken bones, but aesthetically and functionally recover these individuals to return them to their socio-economic development. In this context, blindness fits in as a serious complication that can lead to permanent incapacity. Some injuries that lead to blindness can be completely reversible if diagnosed and treated promptly [23].

Some factors are responsible for the increased incidence of blindness resulting from facial trauma. Over the past four decades, there has been an increasing number of automobile accidents and urban violence. There is a strong predominance of males aged 20 to 40 years who have a significant sight impairment as a result of facial fractures [7]. This fact is related to males because there is a greater number of male drivers and this is associated with a more aggressive behavior on the part of said male drivers. In addition, The auto industry has developed even more powerful, faster vehicles and when these are involved in accidents, the resulting higher impact forces greatly account for the occurrence of eye injuries [19, 20].

Thus, once assessed the immediate needs of polytrauma patients, a careful inspection of the eye should be conducted in order to look for injuries that can lead to loss of vision. Traffic accidents tend to cause fractures in the middle third portion of the face, such as the maxillary Le Fort II and III, and zigomaticorbitary fractures, which in turn can lead to blindness [19].

A thorough eye examination should be performed in all patients who sustain a facial trauma. Examining pupil size may indicate a significant obstruction of the sensory pathway and is extremely useful in the stratification of injuries, and may indicate more serious pathology such as retinal detachment, posterior eyeball rupture or optic nerve damage [7].

Some of the most important actions for the diagnosis and correct care to the patient include evaluating visual acuity, light perception, gross eyeball displacement, fundoscopy, edema or retinal detachment, edema or paleness of the optic disc, constriction of retina arterioles, and venous congestion [9].

The clinical examination of a patient with probable eye-related injury can detect a decrease in visual acuity with relative afferent pupillary defect (Marcus Gunn pupil) and increased intraocular pressure. The Marcus Gunn pupil indicates homolateral afferent arm injury in the optic nerve under light stimulation. The test consists of applying a light stimulus in the pupils alternately in 5second intervals. As an example, a patient with an injury on the right optic nerve will show isocoria to eye opening examination. The direct fotomotor reflection of the right eye will be quite reduced, as well as the fotomotor consensus of the left eye. The fotomotor stimulation of the left eye will provide normal results. Thus, when focusing a light source on the right pupil, both pupils will contract slightly. If the light is directed to the left eye, both will close normally. Repeating again and again to the stimulation of one eye and the other in a given time under stimulation of the affected eye, the pupil will dilate and the expected response would be a contraction of it. This altered fotomotor reflex in the pupil is called Marcus Gunn pupil. Signs often include limited eye movements and proptosis.

Fundoscopy is the most frequently used test to investigate further intraocular lesions, which may reveal optic disc edema, retinal venous congestion, pulsations or occlusion of central retinal artery or retinal edema [24].

Complementary exams are very important to obtain a more accurate diagnosis. Among such exams, the CT scan can be used to evaluate the occurrence of orbital fractures, the location and source of tensions generated from orbit such as RBH, emphysema or foreign body. It has the advantage of being performed quickly and being available in most trauma hospitals. Magnetic resonance imaging has a more limited applicability in acute trauma, as it takes longer to be performed and also requires greater patient cooperation. It is useful in identifying different stages of hemorrhage and showing acute bleeding. MRI is contraindicated in cases of metallic foreign body because its magnetic field can cause displacement of it [6].

Blindness can develop after facial trauma through different mechanisms, but which can be grouped into two major groups: the first involves injuries to the eyeball and the second is related to optic nerve injury [10].

Those injuries of the eyeball can be open-type or closed-type, and result from direct or indirect trauma, which is capable of causing continuity solution of its structure. In open-type injuries, reduction of visual acuity is inevitable and treatment aims to reduce the chances of infection and minimize complications such as blindness and atrophy of the eyeball on the unaffected side due to uveitis. The closed-type injuries are more difficult to diagnose and require specific diagnostic tests such as eye ultrasound. Eye ultrasound has been an important complementary test in the detection of foreign bodies, laceration and detached retina. The prognosis will depend on the level of initial injury and the possible development of post-treatment complications [7,10].

The treatment of eyeball injuries will vary according 
to open-type and closed-type injuries. Preliminary treatment with analgesic and antipyretic drug should be conducted. In open-type injuries, surgical repair should be performed within 24 hours after trauma. At surgery, foreign bodies should be removed and the wound explored in all its extension and then closed with nonabsorbable suture. Antibiotics should be administered to prevent endophthalmitis. Posterior vitrectomy is used to prevent retinal detachment $[11,12]$.

Injuries related to the optic nerve include direct trauma that compromises its anatomic integrity or result from a series of events that will characterize the OCS. The OCS comprises a set of injuries whose pathophysiology is the increased intra-orbital pressure, vascular congestion and subsequent ischemia, with subsequent degeneration of the optic nerve [23].

The optic nerve can be damaged by section, contusion or increased orbital pressure. Most injuries of the optic nerve are related to damage following the intracanalicular section of the nerve; however, displaced bone fragments in any part of the orbital area may also injure it. Clinical findings that suggest an optic nerve damage include decreased visual acuity and a relative afferent pupillary defect.

Clinical evaluation of pupil size and reactivity to light is essential for the diagnosis; the presence of the pupil Marcus Gunn suggests that damage to the optic nerve. The reaction of the slow contraction of the pupil is the first sign of a progressive injury of the optic nerve [10, 21].

The injuries that directly affect the optic nerve are often caused by firearm injuries, or penetrating injuries such as those caused by stab wounds or foreign bodies. In this situation, the tendency is that the damage is ireversible.

Indirect injuries occur when the optic nerve is placed under stress and these can cause lacerations, stretch or break their nerve fibers. The treatment is clinical through prescription of anti-inflammatory, antibiotic and antihypertensive eye drops. The prognosis is grim and will depend on the intensity of the injuries. A long time is required for nerve recovery and it might take one year. From that moment on, any improvement in visual acuity is unlikely [8].

Major injuries can affect the optic nerve as a result of ischemic phenomena in the nerve pathway from the canal to the eyeball. The optic nerve has blood supply from a delicate vascular system that surrounds the nerve sheath. The interruption of blood flow from that system leads to hypoxia of the distal segment of the optic nerve from the injury site. Sixty minutes of hypoxia are sufficient to cause irreversible damage and permanent visual damage, such as the OCS [16].

The compartment syndromes are characterized by in- creased blood pressure in a particular anatomical region that most commonly affects the upper limbs and lower abdomen. This condition causes a collapse in local blood flow, leading to a condition of emergency in which immediate treatment is necessary. The OCS is a less known variant of these syndromes, whose outcome will lead to loss of sight in the affected eye [6].

OCS leads to an increased orbital pressure because the orbit is an enclosure with limited capacity to expand. The diagnosis must be fast and accurate, and be based on clinical exams and imaging such as CT scan and magnetic resonance imaging to facilitate a correct diagnosis of OCS. In acute traumas, the two main phenomena that can cause OCS are RBH and TON [6,10].

$\mathrm{RBH}$ results from bleeding within the orbit that may be located in the intraconal or extraconal space or in both. They occur in most cases as a result of fractures of the orbital walls with small displacements. Slightly displaced fractures of the orbital walls can affect major vessels, which can present significant bleeding and prevent the drainage of blood to other compartments such as the maxillary and ethmoid sinus [15].

Clinically, RBH might be presented with one or more of the following signs: exophthalmos, anisocoria, ophthalmoplegia, relative afferent pupillary defect and paleness of the optic disc. In conscious patients, a reduction of visual acuity may be present or be established progressively [15-17].

CT scan aids in diagnosis and is preferably used than MRI because it can be conducted more quickly. Their findings may demonstrate the presence of a localized collection of blood or mixed blood and fluids infiltrate resulting from diffuse edema in the posterior orbital compartment [14].

The diagnosis is mainly clinical and appropriate treatment should be established whenever a strong suspicion is present, even if it is impossible to conduct imaging studies or when they do not show the characteristic findings [15].

$\mathrm{RBH}$ treatment is surgical and can be divided into primary, with lateral canthotomy, and final, through surgical decompression whereby drainage of the hematoma and hemostasis of bleeding vessels, with the possible installation of a local drain for any residual bleeding, can be conducted. Lateral canthotomy allows for indirect decompression by allowing for a displacement of the eyeball and a consequent increase in the orbital compartment. It is a primary technique because it can be easily performed under local anesthesia, but does not promote hemostasis; therefore, it has no effect on injured vessels nor drains any secretion. It has clinical applicability in emergency situations until the patient is stabilized and can be subjected to definitive treatment with surgical decompression under general anesthesia. Drug therapy 
should be employed with the use of anti-inflammatory steroids in high doses in all cases, unless there is some relevant contraindication [24].

TON is a type of ischemic injury of the optic nerve of traumatic origin in which the major damage will occur very close to the optic nerve with involvement of the microcirculation of its nutrient vessels. A congestion of blood vessels of the neural sheath associated with the edema from chemical mediators, which together undermine the local circulation causing vasospasm and vasoocclusion [8].

The clinical aspects of TON may be similar to those of $\mathrm{RBH}$, but with less intensity. The main TON signs include the reduction of visual acuity and the presence of a relative afferent pupillary defect in the presence of a normal fundoscopy. Generally, its etiology is related to an injury to the optic canal, resulting in nerve damage, or injury caused by displaced bone fragments near the optic nerve [21].

Unlike the RBH, the CT findings are extremely important to support the clinical diagnosis and also clarify the type and location of the injury found. Careful evaluation of the optic canal is paramount and may show fractures and canal narrowing. The presence of bone fragments or foreign bodies in the vicinity of nerve injury suggest a local injury when associated with clinical findings. Finally, thickening of the optic nerve when compared to the unaffected side is suggestive of edema and bleeding in the nerve sheath [17].

The treatment of TON is controversial and may be either clinical or surgical. The group of international studies on optic nerve trauma has tried to establish the ideal treatment for TON using a randomized clinical trial. The groups were divided into those who underwent surgical decompression with high doses of corticosteroids, and the second treated only with corticosteroids. However, after two years of study, the methodology proved to be statistically invalid as to the selection of patients [8]. Therefore, meta-analysis studies show similar results for the treatment, whether medical or surgical. Nevertheless, any treatment is more effective than the none at all [14].

Surgical decompression of TON consists of the removal of foreign bodies that are close to the optic nerve or an endoscopic decompression of the optic canal. Because TON has similar results as to the selection of treatment, unless an evident injury is present and by itself indicates a surgical treatment, clinical therapy should be the first option, as it combines the advantages of presenting results in terms of final visual acuity being similar to a surgical procedure, and it is a noninvasive procedure. Surgical decompression should be performed in cases unresponsive to medical therapy [14].

The inability to close the eyelids is not a common injury to cause blindness, but often leads to discomfort and pain because of cornea dryness due to lack of appropriate lubrication. It can evolve rapidly into ulceration and blindness. Treatment consists of eyelid reconstruction wherever possible; alternatives such as gradual traction of the lacerated eyelid until it covers the cornea, may be necessary. The use of a traction suture can avoid epithelial defects that can evolve with ulceration and blindness. The use of artificial tears and chloramphenicol may be useful [10].

\section{CONCLUSIONS}

In this review, we could observe that blindness is a serious complication of facial trauma and which tends to be irreversible in injuries of the eyeball and in direct injuries of the optic nerve, but may be reversible in OCS, time being a key factor in the prognosis.

In cases of RBH and TON, the diagnosis is simple and easily obtained in a clinical evaluation, with the reduction of visual acuity and the presence of an afferent pupillary defect being the most common characteristics. The treatment of these injuries can be performed on the patient without significant risk of death.

Thus, the evaluation of the eyeball and mainly of the visual acuity should be included in the initial care of trauma patients, so that injuries that can lead to blindness are diagnosed and treated early.

\section{REFERENCES}

[1] Alves, M.R., José, N.K., Prado-Júnior, J., Usuba, F.S., Onclinx, T.M. and Marantes, C.R. (1995) Ferimento perfurante ocular: 400 casos admitidos na clínica oftalmológica do Hospital das Clínicas da Faculdade de Medicina da Universidade de São Paulo. Arquivos Brasileiros de Oftalmologia, 58, 342-345 (in Portuguese).

[2] Voughan, D., Asbury, T. and Riordoun-Eva, P. (2003) Oftalmologia geral. 15th Edition, Atheneu, São Paulo (in Portuguese).

[3] Dados do Censo 2000-IBGE (2002) Instituto Brasileiro de geografia e estatística. IBGE, Rio de Janeiro (in Portuguese).

http://www.ibge.gov.br/home/presidencia/noticias/noticia _impressao.php?id_noticia=438

[4] Whitcher, J.P., Srinivasan, M. and Upadhyay, M.P. (2001) Corneal blindness: A global perspective. Bulletin of the World Health Organization, 79, 214-221.

[5] Brandt, M.T. and Haug, R.H. (2001) Traumatic hyphema: A comprehensive review. Journal of Oral and Maxillofacial Surgery, 59, 1462-1470. doi:10.1053/joms.2001.28284

[6] Lima, V., Burt, B., Leibovitch, I., Prabhakaran, V., Goldberg, R.A. and Selva, D. (2009) Orbital compartment syndrome: The ophthalmic surgical emergency. Survey of Ophthalmology, 54, 441-449.

doi:10.1016/j.survophthal.2009.04.005 
[7] Lindfield, D. and Das-Bhaumik, R. (2009) Emergency department management of penetrating eye injuries. International Emergency Nursing, 17, 155-160.

[8] Levin, L.A., Beck, R.W., Joseph, M.P., Seiff, S. and Kraker, R. (1999) The treatment of traumatic optic neuropathy: The international optic nerve trauma study. Ophthalmology, 106, 1268-1277. doi:10.1016/S0161-6420(99)00707-1

[9] Perry, M., Dancey, A., Mireskandari, K., Oakley, P., Davies, S. and Cameron, M. (2005) Emergency care in facial trauma-A maxillofacial and ophthalmic perspective. Injury, 36, 875-896. doi:10.1016/j.injury.2004.09.018

[10] Smerdon. D. (2000) Anatomy of the eye and orbit. Current Anaesthesia \& Critical Care, 11, 286-292. doi:10.1054/cacc.2000.0296

[11] Jayaram, H. and Calder, I. (2004) Eye and orbit. Anaesthesia \& Intensive Care Medicine, 5, 300-301. doi:10.1383/anes.5.9.300.49895

[12] Wichmann, W. and Muller-Forell, W. (2004) Anatomy of the visual system. European Journal of Radiology, 49, 830. doi:10.1016/j.ejrad.2003.11.001

[13] Schor, P., Belfort Júnior, R. and Chamon, W. (2004) Oftalmologia. Manole, Baueri (in Portuguese).

[14] Plaper, R. (2006) Conhecendo o olho. Manole, Barueri (in Portuguese).

[15] Burkat, C.N. and Lemke, B.N. (2005) Anatomy of the orbit and its related structures. Otolaryngologic Clinics of North America, 38, 825-856. doi:10.1016/j.otc.2005.03.017

[16] Reymond, J., Kwiatkowski, J. and Wysocki, J. (2008) Clinical anatomy of the superior orbital fissure and orbital apex. Journal of Cranio-Maxillofacial Surgery, 36, 346-353. doi:10.1016/j.jcms.2008.02.004

[17] Ansari, M.H. (2005) Blindness after facial fractures: A 19-year retrospective study. Journal of Oral and Maxil- lofacial Surgery, 63, 229-237. doi:10.1016/j.joms.2004.05.221

[18] Perunovic, B., Quilty, R.D., Athanasiou, A. and Love, S. (2001) Damage to intracranial optic pathways in fatal closed head injury in man. Journal of the Neurological Sciences, 185, 55-62. doi:10.1016/S0022-510X(01)00463-4

[19] Turbin, R.E., Maxwell, D.N., Langer, P.D., et al. (2006) Patterns of transorbital intracranial injury: A review and comparison of occult and non-occult cases. Survey of Ophthalmology, 51, 449-460. doi:10.1016/j.survophthal.2006.06.008

[20] Entezari, M., Rabei, H.M., Badalabadi, M.M. and Mohebbi, M. (2006) Visual outcome and ocular survival in open-globe injuries. Injury, 37, 633-637. doi:10.1016/j.injury.2006.02.043

[21] Chen, C., Selva, D., Floreani, S. and Wormald, P.J. (2006) Endoscopic optic nerve decompression for traumatic optic neuropathy: An alternative. Otolaryngology-Head and Neck Surgery, 135, 155-157. doi:10.1016/j.otohns.2005.03.056

[22] Gerbino, G., Ramieri, G.A. and Nasi, A. (2005) Diagnosis and treatment of retrobulbar haematomas following blunt orbital trauma: A description of eight cases. International Journal of Oral and Maxillofacial Surgery, 34 127-131. doi:10.1016/j.ijom.2004.05.001

[23] Perry, M. (2008) Acute proptosis in trauma: Retrobulbar hemorrhage or orbital compartment syndrome-does it really matter. International Journal of Oral and Maxillofacial Surgery, 66, 1913-1920. doi:10.1016/j.joms.2008.04.012

[24] Hislop, W.S., Dutton, G.N. and Douglas, P.S. (1996) Treatment of retrobulbar haemorrhage in accident and emergency departments. British Journal of Oral and Maxillofacial Surgery, 34, 289-292. doi:10.1016/S0266-4356(96)90004-2 